Instituto Internacional de Investigación y Desarrollo Tecnológico Educativo INDTEC, C.A.

DOI: https://doi.org/10.29394/scientific.issn.2542-2987.2017.2.3.4.76-98

OAI-PMH: http://www.indteca.com/ojs/index.php/Revista Scientific/oai

\title{
Los Valores Sociales desde las Voces de los Actores Socio-Educativos
}

Autores: Ana Liseth Rondón Domínguez

Universidad Nacional Experimental Rafael María Baralt, UNERMB

lisethanarondon297@hotmail.com

Nereida Nair Peña Lara

Universidad Nacional Experimental Rafael María Baralt, UNERMB

nere nair3011@hotmail.com

Gaby Angélica Terán Viera

Universidad Nacional Experimental Rafael María Baralt, UNERMB

terangaby2012@gmail.com

Trujillo, Venezuela

\section{Resumen}

El objetivo de esta investigación fue: Develar los valores sociales desde las voces de los actores socio-educativos Para efecto del mismo se abordó desde la investigación cualitativa, que permite conocer los hechos desde la misma realdad, por lo que se asumió el método fenomenológico, a través de la entrevista a 5 docentes y 5 representantes más un representante del Consejo comunal. La entrevista abierta, se denominó entrevista conversacional, permitiendo a los actores socio-educativos conversar con mayor libertad. Entre las categorías emergidas sobre los valores sociales desde las voces de los participantes, fueron: esencia y virtudes de vida, se enseñan y practican, son creencias, costumbres y conductas, son guía. Desde estas categorías se puede decir que los valores sociales llevan a un accionar en un contexto humanístico desde la relación escuela-familia-comunidad y estado. De igual manera, emergieron como categorías: el respeto, el diálogo, solidaridad, responsabilidad y honestidad. Así mismo, requieren que se pongan práctica valores como el respeto, el diálogo, la solidaridad, la honestidad, entre otros. Por lo que se recomienda a los docentes, la familia y comunidad fortalecer los valores desde una epistemología axiológica, crítica, reflexiva que contribuyan a la formación de mejores ciudadanos.

Palabras claves: valores; valores sociales; actores socio-educativos; cualitativo. 


\title{
Social Values from the Voices of Socio-Educational Actors
}

\begin{abstract}
The objective of this purpose of this researching was: unveil the Social Values taking in account the voices of socio-educational actors in this case; it was from the qualitative research, which allows knowing the facts from the same context of the reality. So it was assumed the phenomenological method through an Interview of: five teachers and 5 Representatives, followed by one representative of communal council. It was also used an open interview which is called conversational interview, allowing socio-educational actors free conversations. Among the findings about the social values from the voices of the social actors, it was revealed the essence and virtues of life that can be tough and practice. They also are beliefs, customs and behaviors, they are considered a guide. From these categories it can say that the social valves lead to take actions in a humanistic context since the relationship between school, family-community and state. In the same way, they were produced like categories: the respect, the dialogue, the solidarity, responsibility y honesty. So it requires that the values must be put in practice. Finally, with this study, it recommend that teachers, educational institute, the family and community strengthen the Values from A reflective axiological epistemology, criticism, which contribute to the formation of better citizens.
\end{abstract}

Keywords: vales; social values; socio-educational actors; qualitative. 


\section{Introducción}

Ante los cambios socio-políticos que estamos viviendo, se hace necesario direccionar y enfatizar la necesidad de fortalecer los valores; los cuales constituyen un tema que ha venido preocupando a los seres humanos desde el mismo inicio de la historia. Muchos autores coinciden en que los valores no son fácilmente definibles, ya que tienen diferentes significados según el contexto en que se piensen. En una concepción naturalista se puede decir que éstos tienden a preservar la supervivencia humana. Una, entre muchas definiciones que hablan de los valores, según Smith (1977; citado en Monserrat; 2002:14). como: "algo libremente elegido de entre alternativas después de considerar las consecuencias de cada alternativa, que es actuado repetidamente hasta convertirse en patrón de conducta, que da dirección y significado a la vida".

Entendiendo que los valores están siempre presentes en el ser humano como uno de los principios que rigen los comportamientos humanos y dirigen las aspiraciones de los individuos, o incluso de las sociedades, en pro de su perfeccionamiento o realización. Desde lo señalado cabe hacer énfasis en la necesidad de estudiarlos desde los valores desde una postura humanista e integradora; es decir desde los valores sociales, los cuales vienen a constituir el componente principal para mantener buenas relaciones sociales.

Por ello, se hace necesaria la formación en valores para que los mismos pervivan y se solidifiquen en las relaciones sociales, desde una perspectiva de la cohesión e integración en la convivencia. Los valores sociales sirven de guía para poder convivir sobre la base de la sinergia social, y son la condición que forma y distingue a una comunidad. No obstante, todo esto depende del grupo social en cuestión, se concede más relevancia a unos valores que a otros, a pesar de que ellos existen relaciones e interdependencia.

Sin embargo, según las últimas tendencias se pretende formar a las personas en valores sociales reconociendo la familia como pilar fundamental 
que fortalezca los valores sociales a partir del hogar practicándolos a diario con los miembros del contexto familiar y social. De igual manera, desde la relación y formación que ofrece la escuela como contexto formador, donde deben prevalecer los valores sociales para formar al niño, niña y joven del hoy y mañana.

Cabe referir estudios como el de Ramos (2009), quien señala resultados satisfactorios en cuanto a la formación de valores en el campo educativo que le sirvió a las personas con diversos valores que están presentes en las vidas de los seres humanos. Por otra parte, Chacón (2008) en su estudio presentó los resultados obtenidos fundamentados en la formación de los valores morales desde su enfoque humanista con una responsabilidad social de educar, formar a la familia y sociedad con un sistema de valores sociales donde logró transformar a jóvenes y comunidad en general.

Desde los estudios presentados, se hace necesario señalar que en la Unidad Educativa "El Cumbe", Municipio Valera, Estado Trujillo, se ha venido observando que muchas veces no se establecen los valores sociales necesarios entre los actores de la escuela y comunidad, lo que puede estar afectando de alguna manera el desenvolvimiento tanto de los estudiantes como de los actores que hacen vida en la institución. Debido a lo expuesto, el objetivo de esta investigación fue: Develar los valores desde las voces de los actores socio-educativos. Estudio que se justifica, por ser parte de una investigación en estudio desde el enfoque cualitativo, direccionada por la investigación fenomenológica, para poder develar desde sus percepciones lo que son para ellos y cómo han vivenciado los valores entre la escuela y la familia, considerando que se hacen presentes en todo momento, como seres humanos que interactuamos constantemente. 


\section{Fundamentación Teórica}

\subsection{Los Valores}

Para referir los valores, se hace necesario comenzar a señalar la axiología como la disciplina filosófica que se ocupa del problema del valor. Etimológicamente el término Axiología proviene de dos vocablos griegos: axios que significa valioso, estimable, digno de ser honrado", y "logos" que significa "estudio, tratado o ciencia". Es decir, el estudio de los valores. Para Cardona (2000), la axiología es la disciplina que se encarga del estudio formal de los valores, teniendo hasta hoy día una cantidad de obstáculos derivados principalmente de la ambigüedad con que se ha manejado el concepto de valor, desde sus diversas concepciones, dificultades para medirlos, entre otros. Desde una postura humanista la teoría de la axiológica aparece como la ciencia o filosofía práctica a los llamados problemas de valor, considerando el proceso de valorización como la condición necesaria para que algo se convierta en valor en el que se obtiene de su ontología, en la esencia de su propio ser extenso e intrínseco (Zuleta, 2007: 61).

Desde una perspectiva epistemológica el humanismo se inserta dentro de las orientaciones filosóficas que se han preocupado por entender la naturaleza y la existencia humana, que comenzaron por constatar la existencia de los valores en su análisis filosófico. Para comprender el concepto de valores se halla de la naturaleza inacabada del ser humano, es decir, su indeterminación, pues la persona no está programada, si no que se ve obligada a decidir continuamente, cómo o hacia donde quiere dirigir su existencia. En ese contexto, según Pestaña (2004): los valores son definidos como un deber ser, un ideal, algo que tiende a darse en la naturaleza, pero no se da. Es decir, los valores se conciben como un atributo a lograr constructo referencial que sirve de orientación a la sociedad.

Desde esta perspectiva, los valores pueden determinar la conducta y el comportamiento de las personas, orientar su vida para marcar su 
personalidad. Cabe por otra parte, destacar la relación existente entre los valores y el proyecto de vida, pues para ser humano vale y es, en consecuencia, un valor aquello que desea y que busca en función de sus necesidades. De los valores surgen aspiraciones, objetivos, metas, tanto en lo personal como en lo familiar y en lo profesional; su cobertura puede abarcar mayores ámbitos.

De allí, que los valores tienen íntima relación con la moral, y si se pueden medir sus componentes se evidenciaría que estos tienen elementos morales bien definidos que le dan fuerza, contenido a su significación. Desde esta perspectiva, se justifica la formación de los valores en el individuo, en la medida que vayan siendo capaces de formarse su propia concepción de la realidad que lo determina, lograrán interactuar con un mayor grado de autonomía, asumiendo, por supuesto, los valores de respeto, convivencia, disciplina individual y colectiva.

\subsection{Valores sociales}

Los valores sociales son compartidos por la sociedad que lo genera tiene mucha relación con los valores personales y familiares. Estos valores pueden cambiar de una sociedad a otra; es decir, un valor que en nuestra sociedad venezolana es muy importante, quizás no lo sea para otra sociedad. En referencia, Bello (2004: 32): señala que los valores sociales comienzan a formarse en la práctica como un ejercicio individual, esta constituye para cada individuo un compromiso como alguien que actúa en una comunidad de individuos, que desea resultados positivos en su crecimiento personal y su dimensión social. Los valores sociales, según el Ministerio del Poder Popular para la Educación (2007) indica que se forman en una comunidad específica, en cada población y país, como fruto de un proceso de intercambio.

Los valores sociales provienen de la interrelación entre los miembros de la sociedad por lo que se hace necesario promover lo más adecuado a los 
intereses comunes, los que van a dar la mayor suma de la felicidad a todos. Pero a la vez, es fundamental que se respeten los valores de las minorías y de los individuos, porque todos los constituye una sociedad. Se trata del deber ser para beneficio del colectivo y así una sociedad más humanista.

Por ello, si los actores de la escuela y la familia formamos desde la práctica los valores, donde cada uno se responsabilice de sus acciones, giren en torno al bien común y todos mantenemos una coherencia entre nuestra vida y nuestros valores, estaremos construyendo a la constitución sana de una sociedad de valores. Cabe destacar, que los valores sociales surgen primordialmente en el individuo por influjo y en el seno de la familia y entre los cuales se debe tener en cuenta el respeto, la tolerancia, la honestidad, lealtad, trabajo, y la responsabilidad.

\subsection{Los valores desde una perspectiva socio-educativa}

Ubicando a los valores desde una perspectiva educativa, los mismos vienen a representar un intento de clarificación del distinto teórico sobre el valor y al mismo tiempo una praxis distinta de la educación en valores. Recoge elementos de nuestra esencia del ser humano: valores y educación. Mientras que la preocupación por los valores quedó plenamente asumida en el informe de la Comisión Internacional de Educación para la UNESCO, coordinada por Delors (1996), que tuvo la virtud de recoger las aspiraciones de numerosos colectivos de educadores del mundo y de pensadores que han brillado sus pedagogías, en el que ponen manifiesto los cambios profundos educativos en la transversalidad del desarrollo humano en las sociedades.

Desde un punto de vista socio- educativo, los valores se consideran a referentes pautas o abstracciones que orientan al comportamiento humano hacia la transformación social y la realización de la persona, para ello presenta guías que dan determinada orientación a la conducta y a la vida de cada individuo de cada grupo social (Bello; 2008: 33). Los valores humanos, 
establecen un modelo de conducta que es aceptable en la sociedad; $y$ ayudan a construir comportamientos que lleven a la transformación del ser en ese convivir en los diferentes contextos de su entorno social (Matos, 2000: 25). Desde aquí, radican los criterios y la práctica de valores en el que se identifica el individuo de estar en el mundo del grupo al que pertenece, afirmando una concepción de la humanidad.

\section{Metodología}

Considerando el propósito del estudio, el mismo se abordó desde el paradigma cualitativo, el cual para Pestaña (2004), consiste en descripciones detalladas de situaciones, eventos, personas, interacciones y comportamientos que son observables. Además, incorpora las expresiones de los participantes, sus experiencias, actitudes, creencias, pensamientos, reflexiones, tal como son expresados por ellos mismos. Partiendo de la necesidad de conocer desde las voces de los actores socio-educativos, el estudio se aborda desde una filosofía fenomenológica-hermenéutica procede a la búsqueda de la percepción y descripción de las experiencias de los sujetos y los significados de las mismas; por esta razón, la escogencia del método debe organizarse congruentemente con este enfoque para que evidencie la experiencia del objeto de estudio para buscar el sentido oculto y la esencia de los fenómenos.

El método fenomenológico, parafraseando a Pestaña (2004), permite estudiar los fenómenos como son experimentados y percibidos por el hombre, permitiendo estudiar la realidad desde la naturaleza que sólo puede ser captada desde lo interno del sujeto, quien es el que las vive y la experimenta. Por tal razón, el asumir la postura epistemológica de este estudio desde la fenomenología parte del hecho que la realidad es construida desde los propios actores socio-educativos quienes expresan mediante la reflexión, la percepción que le dan a los valores sociales, considerando que desde la 
realidad planteada no se llevan establecen relaciones sociales acordes y amenas para el buen desarrollo educativo.

Para efecto del mismo, se realizaron entrevistas cualitativas, que de acuerdo con Gurdián-Fernández (2007: 197), es una técnica que, conducida con naturalidad, permitiendo al entrevistado reflexionar sobre su ser, hacer y saber. Existen diferentes tipos de entrevista de acuerdo al contexto de estudio, por lo que para efecto de trabajar con los actores de la escuela y comunidad se realizaron entrevistas más de tipo conversacional, permitiendo a los actores participantes sentirse en confianza y poder expresarse libremente; es lo que llamaría (Valles; 2007: 37) "entrevista conversacional", no queriendo decir con esto que se pierde el valor que implica la entrevista en su profundidad y cientificidad, sino más bien, en el acercamiento y confianza con el entrevistado.

Los actores claves la conformaron 5 docentes (representados por el código Doc desde el 1...5) y 5 representantes (representados por el código Rep 1...5), y un Representante del Consejo Comunal, quienes de alguna manera son los que más hacen referencia a la situación problema en la escuela y comunidad. Entrevista que fue sistematizada y organizada para extraer las categorías emergentes desde las percepciones de los propios actores. Categorías que se presentan como resultado del análisis e interpretación de la información recolectada desde las voces de los participantes claves.

\section{Categorías emergidas}

Entre las categorías emergidas sobre los valores sociales, a continuación, se presentan las siguientes: 


\subsection{Los valores como esencia y virtudes de la vida de la vida}

Desde la postura de Aristóteles los valores son esenciales para la vida, dándole funcionalidad a las cosas y, por lo tanto, le dan razón de ser en la discrecionalidad humana. Los valores dependen del punto de vista que los asuman los seres humanos. Desde las voces de Doc3: "los valores son esenciales para la vida de todo ser humano, sin valores no tendríamos cultura y ética", como puede apreciarse la docente considera a los valores esenciales para el proceder del ser humano.

Para el Rep1. Los valores "son virtudes de toda persona que posee según sus principios formados en el hogar y tienen que ver con la ética y la moral". Por lo que para entender los valores como virtudes, Touriñan (2008), se hace necesario considerar los valores como principios éticos, como acciones que se explican en las virtudes y en las actitudes concretas del ser humano. Por otra parte, Rep4. Indicó "para mí los valores están presente en la vida cotidiana, son esenciales en nuestra vida"

Para Ortega y Mínguez (2001: 28), los valores forman parte de la rutina de la existencia del ser humano, de tal modo que constituyen "unos excelentes termómetros que marcan el grado de integración o desintegración que experimentan los diversos grupos sociales". En este sentido se hace necesario descubrir los valores en uno mismo, tomar conciencia de ellos y ver hasta qué punto éstos orientan la propia vida. Para Murcia (2001: 61), el rol que desempeñan los valores en la vida diaria y su necesidad para guiar la conducta del ser humano, se encuentran íntimamente relacionados con la existencia del hombre. Por lo tanto, como esencia de la vida conducen y orientan al ser humano en su accionar consigo mismo y con los otros, conformando virtudes esenciales en la vida diaria, que orientan la conducta del ser humano, por cuanto los mismos se encuentran en cada sujeto. 


\subsection{Los valores se enseñan y practican}

De acuerdo con la Doc2. "Los valores se enseñan en el hogar, pero todos los días debemos practicarlos corrigiendo a nuestros hijos en las cosas malas que hacen se debe corregir al momento no dejar para después". Por su parte, Rep4, señaló "para mí los valores son los que nos enseñan desde nuestros hogares y escuela que debemos practicar día a día".

De acuerdo con Ortega y Mínguez (2001), los valores se pueden enseñar, pero no como cualquier área del conocimiento, tiene características específicas que la distinguen, por lo que refiere que podrían transmitirse en la escuela, con algunas garantías de éxito. Para Carreras, Eijo, Estany, Gómez, Planas y Serrats (2001), la necesidad de valores se justifica por la necesidad que tenemos los individuos de comprometernos con determinados principios éticos que sirvan para evaluar las propias acciones y la de los demás.

De igual manera, un Rep del Consejo Comunal dijo: "Bueno, son todos aquellos que nos inculcaron y que debemos poner en practicar en la comunidad". Para Doc2 "Son los valores que aprendemos a compartir, convivir con un grupo de personas dentro y fuera de una comunidad". Por lo que Carreras y otros (2001: 14), señalan que "los valores son contenidos que pueden ser aprendidos y pueden ser enseñados". La educación en valores debe hacerse con docentes, padres y representantes durante las reuniones, que los lleve a reflexionar sobre el acto de valorar y ponerlos en práctica en la vida cotidiana.

\subsection{Los valores son creencias, costumbres y conductas}

Constituye otra de las categorías, cuando la Doc4: señaló "Los valores son creencias, costumbres y actitudes de conductas o comportamientos del individuo, ellos están presentes en todo momento del día, pero en nuestra sociedad vemos cosas negativas que quizás se le presta más atención, que a las cosas positivas que debemos reforzar día a día". Así mismo la Doc3. señaló 
"son costumbres que asumimos los seres humanos para actuar en sociedad, son los que orientan la conducta ante determinada situación". Por lo que Ortega y Mínguez (2001), que los valores morales son creencias o convicciones profundas que guían la existencia humana.

Los valores humanos, establecen un modelo de conducta que es aceptable en la sociedad; y ayudan a construir comportamientos que lleven a la transformación del ser en ese convivir en los diferentes contextos de su entorno social (Matos, 2000. pág. 25). En tal sentido, los valores sirven para guiar las conductas de las personas, son el fundamento por el cual hacemos o dejamos de hacer cosas en un determinado momento. Por lo tanto, los valores guían la conducta del ser humano, siendo necesario fortalecer los valores sociales para un convivir en armonía.

Como costumbres, los valores dependen del contexto donde se desenvuelve el individuo, para Ortega y Mínguez (2001), son aceptados como componentes estables y permanentes en la medida en que se aplique en un determinado contexto. En este sentido los actores sociales lo ven como costumbre, pues en la medida que lo aplica en sus hogares, escuela, comunidad lo ponen en práctica, debido a que es lo que ven.

Como creencias, suponen los actores sociales, Rep5: "para mí son creencias que nos han inculcado nuestros padres y que son necesarios poner en práctica para vivir en sociedad". Por su parte Carreras y otros (2001: 13), señalan que es la "organización de creencias y opciones relativas a diferentes abstractos o principios, normas de comportamiento o modelos, a fines de su vida, que pueden contribuir a convivir en armonía.

\subsection{Los valores son un deber ser}

Los valores desde los actores sociales son un deber ser, cuando en palabras de Doc4. Señala "Ios valores sociales son un deber ser para poder convivir en armonía, donde debe haber respeto, solidaridad y compañerismo". 
Desde la voz de la Docente, se evidencia un deber ser como ciudadanos, que según Pestaña (2004): los valores son un deber ser, un ideal, algo que tiende a darse en la naturaleza, pero no se da. Es decir, los valores se conciben como un atributo.

De igual manera, se aprecia en la expresión la concepción de unos valores sociales que llevan a un convivir en armonía, reflejándose otros valores inmersos como la solidaridad, el respeto, la solidaridad. Desde una postura humanista genera acción, tal como lo refleja la Doc1. "los valores para mí son un deber ser como ciudadanos que debemos operacionalizarlos en el día a día, de nada vale que los conozcamos si no lo practicamos". Expresión que lleva a relacionar los valores con la parte afectiva, actitudinal y conductual, elementos que generan un proceso cognitivo y que se traduce en creencias del ser humano para vivir en sociedad, muy relacionado con posturas anteriores.

\subsection{Los valores como guía}

Los valores como guía, fue otra de las expresiones emergidas en el discurso de Rep del CC, quien señaló: "Ios valores son nuestra guía en el día a día, no sé cómo hay gente que se le hace fácil actuar en contra de ellos", de igual manera Doc3. Indicó: "los valores guían nuestro accionar en cualquier espacio que estemos, por eso es importante que tengamos principios éticos y morales para actuar adecuadamente en sociedad".

Desde las voces de los actores sociales, los valores orientan al comportamiento humano hacia la transformación social y la realización de la persona, para ello presenta guías que dan determinada orientación a la conducta y a la vida de cada individuo de cada grupo social (Bello; 2008: 33). Como puede apreciarse, no sólo se trata del acto valorativo sino también del acto afectivo ante la situación que vive el día a día y que pueden orientar de forma positiva o negativa al ciudadano. 
Además, cuando señala la Doc2 "tengamos principios éticos y morales para actuar en sociedad", está haciendo referencia a lo moral como normas, patrones o conducta, que para Murcía (2001: 64), "conforman las normas o grupo de la sociedad". Es decir, que las normas de conducta están determinadas en gran medida por el ambiente cultural en el que viven. En este sentido Freire (1996: 74), indica que la responsabilidad ética de un profesional de la enseñanza plantea el deber de prepararse, de capacitarse y formarse constantemente, es ser un educador humanista.

Los valores se pueden definir como ideales que siempre hace referencia al ser humano, lo cual los convierte en virtudes personales o cualidades adquiridas, hasta el punto de convertirse en hábito de comportamiento personal y social. Desde esta perspectiva, los valores son vistos más desde una perspectiva social, que pueden determinar la conducta con el comportamiento de las personas, orientar su vida para marcar la personalidad.

\subsection{Los valores sociales están presentes en nuestro accionar}

En un primer momento se definió los valores en forma general, observándose que en su mayoría son más enfatizados desde la relación social, por lo que la investigadora peguntó sobre qué era para ellos los valores sociales, a lo que le respondieron, una Rep4: "Yo opino, que los valores sociales son todos aquellos que están presentes en la familia y comunidad como: el respeto, responsabilidad, cooperación, compartir, amor, amistad, paz, trabajo, apoyo entre vecinos y que muchas veces no son practicados en la comunidad". Por su parte el Rep del C.C: "Para mí son los que prevalece en un contexto sea escuela, familia y comunidad; para poderlos reforzar tenemos que poner en práctica todos los días y tomar todos los valores positivos: solidaridad, compañerismo, amistad, responsabilidad y trabajo en equipo.

Como se puede apreciar es la relación social entre escuela, familia y comunidad que deben ponerse en práctica todos los días, resaltando valores 
como: solidaridad, compañerismo, amistad, responsabilidad y trabajo en equipo, el respeto, responsabilidad, cooperación, compartir, amor, amistad, paz, trabajo, apoyo entre vecinos, entre otros. Cabe destacar que estos son denominados por los actores sociales como valores positivos.

Desde este contexto social, los valores llevan a unavisión ecológica, la cual para Abarca (1996), son entidades socio-culturales de construcción subjetiva originada en las redes relacionales de la persona con el ambiente, que se socializan y se internalizan con rechazo o aceptación relativa, según las características históricas culturales. Se aprecia un carácter relacional teniendo en cuenta la función del ambiente, en tanto mediador o ente al cual se debe adaptar el ser humano.

Así mismo, en expresiones de Doc1: "Los valores sociales son todos aquellos que podemos tener como seres humanos desde la enseñanza de nuestros padres, pero que debemos percibirlos en forma positiva ante una sociedad y reforzarlos, practicarlos para fortalecerlos con los padres, madres, representantes, estudiantes ante la comunidad". R: "Profe, pienso que los valores sociales son los que nos ayuda a vivir en paz con otras personas, mantener buenas relaciones personales con nuestros vecinos, hijos, maestros y familiares":

Desde las expresiones se pueden visualizar diversos elementos implícitos, tales como: percibirlos de forma positiva, practicarlos con los otros, vivir en paz, los cuales son relevantes para fortaleces los espacios escuela y comunidad desde una postura humanística. Tal como lo refiere Doc4: "Es vivir en armonía con un grupo de personas que nos rodea en la escuela y comunidad". Así mismo, el Rep del C.C: "Son todos aquellos valores que prevalece en la comunicación como el amor, paz, responsabilidad y compromiso para vivir en armonía con las personas que nos rodean".

Condición de realización humana que va más allá de la simple normativa que podemos visualizar en la CRBV (1999), en el Diseño curricular, 
entre otros; lo cual permite transformar a los valores desde una postura individualista a una colectiva, más humanista desde los propios actores socioeducativos. Los valores sociales configuran principios para el accionar en conjunto escuela y comunidad y demás espacios donde hacen vida los ciudadanos.

Desde lo develado, se puede decir que los valores sociales son visto como esencia y virtudes de vida, que se pueden enseñar y practicar, son creencias, costumbres y conductas, a la vez guía y, como valores sociales llevan a un accionar en un contexto humanístico desde la relación escuelafamilia-comunidad y estado.

\subsection{Valores que se practican en la escuela y familia}

Ésta categoría surge como parte del proceso que se viene generando en la escuela y comunidad, puesto que una Rep5, dijo "yo enseño a mi niña a respetar a los demás, pero cuando converso con ella de lo que hizo en la escuela me dice, mi amiga gritó muy feo a un compañero y la maestra sólo dijo pórtense bien", expresión que de acuerdo con la representante pareciera que la docente no hizo énfasis en ese momento del respeto por el otro. Por lo que para Yarce (2004) el valor del respeto es ante todo el que nos permite convivir con las demás personas, y hasta con el contexto en el que vivimos es el respeto por la naturaleza, pos sí mismo y por el otro.

Es respeto fue uno de los valores en el que más hicieron referencia los docentes y representantes, desde la valoración entre los mismos docentes, representantes y al estudiantado. Fundamentado en el reconocimiento de la dignidad de cada uno de nosotros a pesar de las diferencias y particularidades. Se trata de fortalecer el respeto desde el mismo respeto pos sí mismo, por los demás y la naturaleza. Están conscientes del valor, sin embargo, en ocasiones pareciera no considerarlo. 
Otro de los valores que emergió desde las voces de los actores socioeducativos fue el Diálogo; al referir una Rep1. "Se requiere de fomentar el diálogo como valor social entre la escuela y la familia, no podemos seguir gritándonos y hasta callando lo que deseamos informar, con respeto se puede establecer un buen dialogo, una buena comunicación". Como puede apreciarse el diálogo es un valor social relevante para los actores socioeducativos, pues como señala López (2005): es un coloquio o conversación con dos o más personas, en relación con los valores que debe fomentarse para intercambiar opiniones, en el que se genere armonía entre las personas del entorno, comunicándose para así interaccionar con opiniones y ayudar a las demás personas en su entorno social.

Así mismo, emerge el valor de la solidaridad, como cultivo de la amistad, pues para la Rep 5, Cuando trabajamos en equipo escuela y comunidad pasamos a fortalecer la solidaridad y con ella la amistad. Para Bello (2004: 77) señala que es colocarnos en el lugar del otro, sentir lo que él sentiría y regresar. De este valor se puede decir, que desde las voces de los actores se pone en práctica cuando se hacen actividades en beneficio del colectivo. Para una Doc4 "debemos tratar de fomentar cada día la solidaridad, lo realizo en el aula y fuera de ella, donde se ponga en práctica el ayudar a las otras personas". Para el REp CC "en su convivir en el hogar y su contexto social, todos podemos fortalecer muchos valores como: la amistad, la confianza, la solidaridad, el trabajo en equipo, el respeto, la ayuda mutua y el reconocimiento del otro".

De igual manera, el valor responsabilidad; prevaleció entre las expresiones de los actores, cuando Rep2, señaló "los maestros nos exigen que seamos responsables, pero ellos no lo son a veces piden tantas tareas a los niños que ni las revisan, para mí eso es ser irresponsable". Sin embargo, una Doc3, dijo "Los padres y representantes muchas veces son 
irresponsables, pues no cumplen con su labor de ser responsable en el acompañamiento de la formación de sus niños y niñas”.

Como se puede apreciar, existe una contradicción entre lo que señalan los padres y los docentes, ya que la responsabilidad es un valor que deben cumplir ambas partes en beneficio de la formación del estudiantado. Sin embargo, Rep2 dice "soy responsable cuando contribuyo con las actividades que la escuela asigna, cuando cumplo con mi compromiso de madre". Para Bello (2008) la responsabilidad es cumplir un trabajo sin presión alguna con las personas, este valor se fomenta en la escuela y la familia al asumir un compromiso y responder a las acciones que están dentro del entorno social. Valor que también permite fortalecer la confianza que viene a contribuir con la amistad, el trabajo compartido, haciendo posible la autenticidad de un proceso educativo sustentado en valores para una mejor educación.

Desde estas percepciones, cabe señalar que hay necesidad de fortalecer los valores desde la propia práctica reflexiva y critica, que lleve a fomentar la honestidad, que de acuerdo con Bello (2008), implica vivir en coherencia con uno mismo y los propios valores. En este sentido se hace presente en los valores que se fomenten y practiquen en la escuela, hogar y en la sociedad.

\section{A manera de reflexión}

Desde lo develado de las voces y percepciones de los actores socio educativos, se puede decir que ellos están claros en lo qué son los valores, su fortalecimiento y la práctica para una mejor sociedad. De igual manera, la necesidad de fortalecer valores de responsabilidad, solidaridad, confianza, respeto, entre otros, que los lleve a convivir en armonía por el bien del estudiantado como de los propios actores que hacen vida dentro y fuera de la escuela.

En este sentido, la escuela y la familia son las encargadas de educar y 
fortalecer valores que contribuyan a crear un clima adecuado dentro y fuera de la institución, en la comunidad y en el hogar, donde prevalezca una comunicación dialógica con éxito, con respeto y de solidaridad. Si bien es cierto, que los padres han de fomentar el intercambio de valores procedentes de sus hogares, también la escuela puede contribuir a favorecer y promover este clima y entorno relacional propicio para una educación de calidad.

Para ello, es importante aprender a convivir en los diferentes espacios en los que nos desenvolvemos, donde padres, docentes y demás actores, no sólo hablemos de la necesidad de una sociedad de valores que la beneficien, sino también que los pongamos en práctica para experimentar la convivencia armoniosa, afectiva, reflexiva, y así avanzar como ser humano con valores dignos y, con ello, fortalecer nuestra sociedad, que hoy día requiere que pongamos todos de nuestra parte para esa mejor sociedad.

\section{Referencias}

Abarca, R. (1996). Vocabulario filosófico científico. Arequipa.

Bello, J. (2004). Valores Esenciales para la vida en familia y en la comunidad. Venezuela: Consejo Nacional de Cultura CONAC.

Bello, J. (2008). Valores para Construir Ética. Venezuela: Melvin.

Cardona, A. (2000). Formación de valores: Teoría, reflexiones y respuestas. México: Grijalbo.

Carreras, LL.; Eijo, P; Estany, A; Gómez, M; Guich, R; Ojeda, M; Planas, T; Serrais, M. (2001). Cómo Educar en Valores (4ta. ed) Madrid: Narcea. Chacón (2008). La formación de valores morales desde su enfoque humanista. Tesis Doctoral presentada en la Comunidad de la Habana Cuba, en la Universidad Pedagógica enrique José Varona.

Constitución de la República Bolivariana de Venezuela (1999). Gaceta Oficial № 5.453 extraordinario. Marzo 24 año 2000. Venezuela.

Delors, J. (1996). La educación encierra un tesoro. España: Santillana. 
Freire, P. (1996). Pedagogia da autonomia: saberes necessários à pratica pedagógica. São Paulo, Brasil: Paz e Terra.

Gurdían-Fernández, A. (2007). EI Paradigma Cualitativo en la Investigación Socio-Educativa. Colección IDER. San José de Costa Rica.

López, H. (2005). Pautas de transmisión de valores en el ámbito familiar. Tesis Doctoral. España: Universidad de Murcia.

Matos, Y. (2000). Una aproximación a la identificación de valores en el proceso enseñanza-aprendizaje de la matemática. Tesis. Realizada en la UNESR, Caracas, Venezuela.

Ministerio del Poder Popular para la Educación (2007). Diseño Curricular del Sistema Educativo Bolivariano. Caracas: Autor.

Montserrat, G. (2002). ¿Qué significa intervenir educativamente en desadaptación social?. España: Horsorsi.

Murcia, J. (2001). Educación en Valores y Derechos Humanos. Colombia: Arfo Editores.

Ortega, R. y Mínguez (2001). Los Valores en la Educación. Editorial Ariel. Pestaña, P. (2004). Aproximación conceptual al mundo de los valores. REICE. Revista Iberoamericana sobre Calidad, Eficacia y Cambio en Educación, 2(2), 67-82, e-ISSN: 1696-4713.

Ramos, A. (2009). Violencia y Victimización en Adolescentes Escolares. Tesis Doctoral. Universidad Pablo de Olavide, Sevilla, España

Touriñan, J. (2008). Educación en valores. Educación intercultural y formación para la convivencia. España: Oleiros. La Coruña.

Valles, M. (2007). Entrevistas cualitativas. Cuadernos metodológicos $N^{\circ} 32$. España: CIS.

Yarce, J. (2004). Valor para vivir los valores: cómo formar a los hijos con solido sentido ético. Bogotá: Norma.

Zuleta (2007). Una Docencia Enjuiciada. Mérida, Venezuela: Consejo de Publicaciones de la Universidad de los Andes. 


\section{Ana Liseth Rondón Domínguez \\ e-mail: lisethanarondon297@hotmail.com}

Trujillo, Venezuela. Estudiante del doctorado en

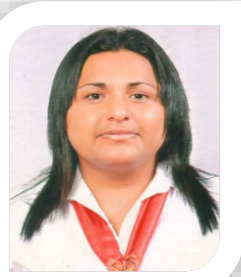

Educación de la Universidad Nacional Experimental "Rafael María Baralt". Laboro actualmente en la Institución: UE "El Cumbe", como docente de aula, Directora y Subdirectora de la Institución. Coordinadora: Responsable del Colectivo de Formación Permanente de Investigación. Profesora del Programa de Formación de Educadores (PNFE), Facilitadora de talleres de convivencia, Paz, educación, Planificación, Investigación Acción, entre otros. Participante Activa de la Línea de Investigación "Investigadores en Acción Social" (IAS) Universidad Nacional experimental Simón Rodríguez, Núcleo Valera. 


\section{Nereida Nair Peña Lara \\ e-mail: nere nair3011@hotmail.com}

Trujillo, Venezuela. Trabajo en la E.B. Monseñor Lucas

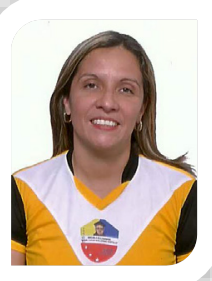

Guillermo Castillo: Estudiante del Doctorado en Educación de la Universidad Nacional Experimental "Rafael María Baralt". Docente desde el 2003 y a partir del 2013 Coordinadora de Protección y Bienestar Estudiantil, en la E.B. Monseñor Lucas Guillermo Castillo. 
Trujillo, Venezuela. Estudiante del Doctorado en

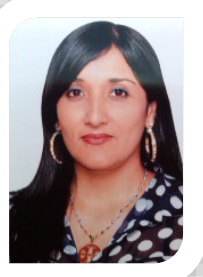
Educación de la Universidad Nacional Experimental "Rafael María Baralt" Msc. en Gerencia de Recursos Humanos. Técnico en Educación Integral. Licda. Ciencia Política. Participante en ponencias a nivel nacional e Internacional, en talleres de formación, facilitadora de talleres. Actualmente docente en el Liceo Antonio Nicolás Briceño. 